\title{
Numerical Solutions of Serrin's Equations by Double Exponential Transformation
}

\author{
By
}

\author{
Shinsuke HAMADA*
}

\begin{abstract}
We consider Serrin's equations, which describe a steady flow of the incompressible viscous fluid caused by an interaction between a vortex filament and a planar wall. They are integro-differential equations with a singularity at an end point. By means of the double exponential transformation, we numerically solve their solutions with high accuracy, and compute a sufficient condition on the uniqueness of the solution.
\end{abstract}

\section{$\S 1 . \quad$ Introduction}

The purpose of the present paper is to numerically solve Serrin's equations:

$$
\begin{aligned}
f^{\prime}(x)+ & f^{2}(x)=k^{2} \frac{G(x)}{\left(1-x^{2}\right)^{2}}, \quad(0 \leq x<1) \\
\Omega^{\prime \prime}(x)+ & 2 f(x) \Omega^{\prime}(x)=0, \quad(0 \leq x<1) \\
G(x)= & 2(1-x)^{2} \int_{0}^{x} \frac{t \Omega^{2}(t)}{\left(1-t^{2}\right)^{2}} d t \\
& +2 x \int_{x}^{1} \frac{\Omega^{2}(t)}{(1+t)^{2}} d t-P\left(x-x^{2}\right), \quad(0 \leq x<1)
\end{aligned}
$$

with the boundary conditions

$$
f(0)=0, \quad \Omega(0)=0, \quad \Omega(1)=1,
$$

where $k \geq 0$ and $P \geq 0$ are parameters, $x \in[0,1)$, and $f, \Omega$ are unknown functions. The prime denotes the differentiation. These equations were derived

Communicated by H. Okamoto. Received September 8, 2006. Revised November 24, 2006.

2000 Mathematics Subject Classification(s): 35Q35, 65R20.

*Research Institute for Mathematical Sciences, Kyoto University, Kyoto 606-8502, Japan. 
by Serrin [9], from the stationary Navier-Stokes equations of incompressible viscous fluid motion to describe an interaction between a vortex filament and a planer wall: Consider the spherical coordinates $(R, \alpha, \theta)$, where $R$ is the distance from the origin, $\alpha$ is the angle between a position vector and the positive $z$-axis, and $\theta$ is the meridian angle about the $z$-axis. The plane $z=$ 0 ( or $\alpha=\pi / 2$ ) is the boundary wall, and a vortex filament is located on the positive $z$-axis, i.e. $\alpha=0$. Accordingly, we consider the Navier-Stokes equations in $R>0,0<\alpha<\pi / 2$ and $0 \leq \theta<2 \pi$. We assume the following conditions: (i) the motion is axisymmetric, (ii) $\boldsymbol{v}=0$ at $z=0$, (iii) $v_{\theta} \rightarrow C / r$ as $\alpha \rightarrow 0$, where $C$ is a prescribed positive constant representing the strength of the vortex filament, and (iv) the vortex filament is neither a sink nor a source. In addition to (i), we assume the following similarity form:

$$
v_{R}=\frac{G(x)}{r}, \quad v_{\alpha}=\frac{F(x)}{r}, \quad v_{\theta}=\frac{\Omega(x)}{r},
$$

where $x=\cos \alpha$ and $r=R \sin \alpha$ with $r$ being the distance from $z$-axis. Setting $F(x)=2 \nu\left(1-x^{2}\right) f(x)$ and some calculations leads to (1.1)-(1.4).

He studied these equations and obtained certain conditions on $k$ and $P$ about the existence and non-existence of solutions. He also obtained some properties of functions $f, \Omega$ and $G$, and computed solutions numerically by successive iterations. Goldshtik \& Shtern [3] studied some equations which were equivalent to Serrin's equations. They derived the asymptotic expansion of solutions and numerically computed solutions by the shooting method. They found two regions of the parameter space $(k, P)$; one is the region where at least one solution exists and another is the subregion of the former where there are at least two solutions.

The right hand side of (1.1) has a singularity at $x=1$, which causes a difficulty in a numerical computation. Serrin showed that $f$ diverged at the rate of $O(|\log (1-x)|)$ as $x \rightarrow 1$ because of the singularity. Thus, this singularity must be taken into account for the computation of Serrin's equations to keep the accuracy of numerical computation. One of the naive methods to eliminate such a singularity is regularization. For fixed $\varepsilon>0$ the equation (1.1) is replaced by

$$
f^{\prime}(x)+f^{2}(x)=k^{2} \frac{G(x)}{\left(1-x^{2}+\varepsilon\right)^{2}} .
$$

Since the right hand side of (1.5) is nonsingular in $[0,1]$, we compute (1.2)(1.5) by a conventional numerical method. By letting $\varepsilon$ tend to zero, we can obtain approximate solutions of Serrin's equations. However, this method has 
a problem. In order to obtain a good accurate solution, we have to make $\varepsilon$ very small. But too small $\varepsilon$ makes equation (1.5) almost singular and we can hardly compute (1.2)-(1.5) with good accuracy.

In this paper, to compute Serrin's equation, we propose a new numerical scheme using the double exponential transformation and the Chebyshev expansion. The double exponential transformation was proposed by Takahasi \& Mori [10] to compute definite integrals with high accuracy. Later, Sugihara [8] rigorously proved optimality of the double exponential formula in a certain class of functions. In other words, he denied the possibility that hyper-double exponential formula like triple exponential formula works better than double exponential formula. Recently, the double exponential transformation is used not only to definite integrals but also to indefinite integrals $[6,11]$, to differential equation [7], and to integral equations [5].

This paper consists of six sections. We propose a numerical method for indefinite integrals using the double exponential transformation and the Chebyshev expansion in Section 2. Using the proposed method, we compute Serrin's equations in Section 3. In Section 4, we check the accuracy of our computation. We examine in Section 5 Serrin's proposition for the existence of solutions in [9] using our method. The conclusion is given in Section 6 .

\section{$\S 2 . \quad$ Numerical Method for Indefinite Integrals}

In what follows, instead of (1.1)-(1.4), we consider the following integral forms:

$$
\begin{aligned}
& \Omega(x)=\frac{\int_{0}^{x} \exp \left(-2 \int_{0}^{t} f(s) d s\right) d t}{\int_{0}^{1} \exp \left(-2 \int_{0}^{t} f(s) d s\right) d t} \\
& f(x)=\int_{0}^{x}\left[-f^{2}(t)+k^{2} \frac{G(t)}{\left(1-t^{2}\right)^{2}}\right] d t .
\end{aligned}
$$

It is easily verified that (1.1)-(1.4) are equivalent to (2.1), (2.2), and (1.3). In order to compute them numerically with high accuracy, we propose a new method using the double exponential transformation and the Chebyshev expansion.

In general, let us consider an indefinite integral

$$
F(x)=\int_{0}^{x} f(t) d t,
$$

where $f$ is analytic in $[0,1)$ and integrable in $[0,1]$. Note that $f$ may have an integrable singularity at $x=1$. We define the double exponential transformation 
$x=\phi(\tau)$ by

$$
\phi(\tau)=\tanh \left(\frac{\pi}{2} \sinh \tau\right), \quad(0 \leq \tau<\infty) .
$$

Using the change of variable $x=\phi(\tau)$, we rewrite (2.3) as

$$
F(\phi(\tau))=\int_{0}^{\tau} f(\phi(s)) \phi^{\prime}(s) d s .
$$

Introducing a large parameter $L>0$, we consider (2.5) in $0 \leq \tau \leq L$. Even if $f(\phi(s))$ tends to infinity, $\left|f(\phi(s)) \phi^{\prime}(s)\right|$ tends to zero rapidly as $s \rightarrow \infty$ because of the very rapid decay of $\phi^{\prime}(s)$. We therefore choose the value of $L$ so that the integrand of (2.5) is effectively zero for $s>L$. Then $\phi(L)$ is very close to one, since $L$ is sufficiently large. Therefore, we have the approximation,

$$
F(1) \approx \int_{0}^{L} f(\phi(s)) \phi^{\prime}(s) d s
$$

with very small error.

We now introduce the change of variable $\tau=L(t+1) / 2$ and set $x(t)=$ $\phi(L(t+1) / 2)$, with which $(2.5)$ is rewritten as

$$
F(x(t))=\int_{-1}^{t} f(x(s)) x^{\prime}(s) d s .
$$

The integrand $f(x(s)) x^{\prime}(s)$ is analytic in $[-1,1]$; thus we can expand the integrand by the Chebyshev polynomials $\left\{T_{j}(x)\right\}_{j=0}^{\infty}$ :

$$
f(x(s)) x^{\prime}(s)=\sum_{j=0}^{\infty} c_{j} T_{j}(s),
$$

where $\left\{c_{j}\right\}_{j=0}^{\infty}$ are determined by

$$
c_{j}=\frac{1}{\lambda_{j}} \int_{-1}^{1} f(x(s)) x^{\prime}(s) T_{j}(s) w(s) d s,
$$

with

$$
\lambda_{j}=\left\{\begin{array}{ll}
\frac{\pi}{2} & j \neq 0, \\
\pi & j=0,
\end{array} \quad w(s)=\frac{1}{\sqrt{1-s^{2}}} .\right.
$$

Substituting (2.8) into (2.7), we have

$$
F(x(t))=\int_{-1}^{t} \sum_{j=0}^{\infty} c_{j} T_{j}(s) d s=\sum_{j=0}^{\infty} c_{j} \int_{-1}^{t} T_{j}(s) d s .
$$


The actual numerical procedure goes as follows: First, noting that $\left\{c_{j}\right\}_{j=0}^{\infty}$ decay exponentially as $j \rightarrow \infty$, we regard $c_{j}$ as approximately zero for sufficiently large $j$. Therefore, $f(x(s)) x^{\prime}(s)$ can be approximated by

$$
f(x(s)) x^{\prime}(s) \approx \sum_{j=0}^{N} c_{j} T_{j}(s),
$$

in which $N=2^{m} \quad(m \in \mathbb{N})$ sufficiently large. Remembering that $(2.11)$ is the Chebyshev interpolation of $f(x(s)) x^{\prime}(s)$, we compute $\left\{c_{j}\right\}_{j=0}^{N}$ by

$$
c_{j} \approx \frac{\nu_{j}}{N} \sum_{k=0}^{N} f\left(x\left(s_{k}\right)\right) x^{\prime}\left(s_{k}\right) T_{j}\left(s_{k}\right),
$$

where $s_{j}=\cos (j \pi / N), \nu_{0}=1$ and $\nu_{j}=2$ for $j \geq 1$, which is computed by the discrete cosine transformation. Next, $F(x(t))$ is approximated by

$$
F(x(t)) \approx \sum_{j=0}^{N} c_{j} \int_{-1}^{t} T_{j}(s) d s .
$$

The integration of $T_{j}(s)$ is evaluated by the following formula:

$$
\int_{-1}^{t} T_{j}(s) d s= \begin{cases}t+1, & j=0 \\ \frac{1}{2}\left(t^{2}-1\right), & j=1 \\ \frac{T_{j+1}(t)-(-1)^{j+1}}{2(j+1)}-\frac{T_{j-1}(t)-(-1)^{j-1}}{2(j-1)}, & j \geq 2 .\end{cases}
$$

To check if the numerical scheme works, we compute the following indefinite integrals:

$$
\begin{array}{ll}
\text { example 1: } & \int_{0}^{x} \log \frac{1}{1-t} d t=(1-x) \log (1-x)+x, \\
\text { example 2: } & \int_{0}^{x} \frac{d t}{\sqrt{1-t^{2}}}=\arcsin x, \\
\text { example 3: } & \int_{0}^{x} \sqrt{1-t^{2}}=\frac{1}{2}\left(\arcsin x+x \sqrt{1-x^{2}}\right), \\
\text { example 4: } & \int_{0}^{x} t d t=\frac{1}{2} x^{2} .
\end{array}
$$

Both of the integrands in examples 1 and 2 have a singularity at $x=1$. In particular, example 1 is important for the computation of Serrin's equations since the integrands of Serrin's equations have the same log-singularity, 


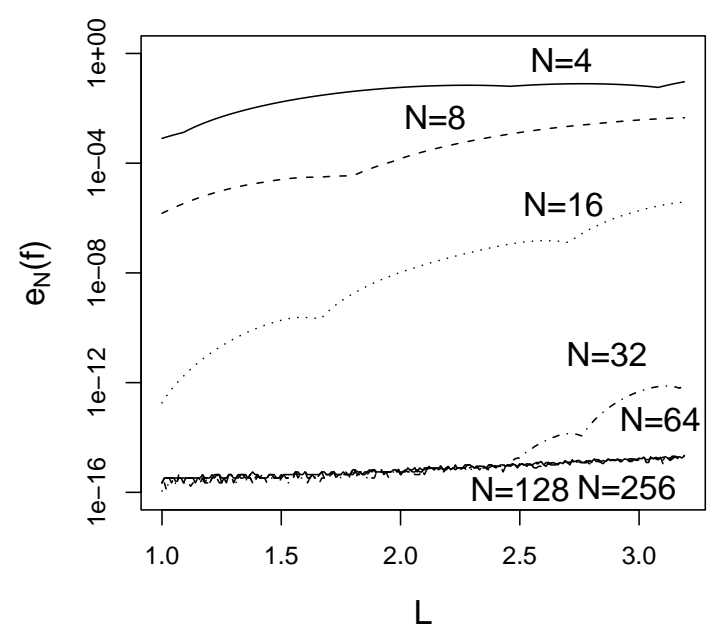

Figure 1. Log plot of the maximum errors for the example 1.

$O\left([\log (1-x)]^{2}\right)$ as $x \rightarrow 1$. The integrand of example 3 is continuous at $x=1$, but the derivative of the integrand has a singularity at $x=1$. Example 4 is the analytic case.

Figures 1-4 show log-plot of the maximum error $e_{N}(f)$, which is defined by

$$
e_{N}(f)=\max _{0 \leq j \leq N}\left|I\left(f ; x_{j}\right)-I_{N}\left(f ; x_{j}\right)\right|,
$$

where $f$ is a integrand, $x_{j}=x\left(s_{j}\right), s_{j}=\cos (j \pi / N)$,

$$
I\left(f ; x_{j}\right)=\int_{0}^{x_{j}} f(x) d x,
$$

and $I_{N}\left(f ; x_{j}\right)$ is the approximation of $I\left(f ; x_{j}\right)$. Our computation was carried out by Pentium-M processor with Windows XP, and the double precision floating-point computation was used. We compute each integrals with $L=1.0$, $1.01, \ldots, 3.19,3.2$ and the number of collocation points $N=4,8,16,32,64$, $128,256$.

From Figures 1-4, we see that the maximum errors rapidly decrease. For example, the error attains the limit of error of the double precision at $N=64$ 


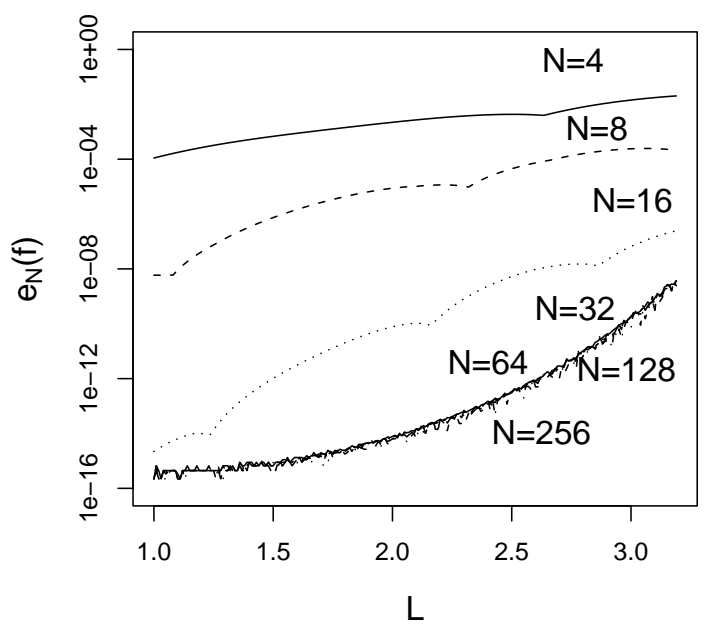

Figure 2. Log plot of the maximum errors for the example 2.

in the example 1. We can also observe almost the same result in the examples 3 and 4 . But in the example 2, the maximum errors do not reach the limit of errors of double precision. Note that in computation of the example 2, we have to compute $1 / \sqrt{1-x^{2}}$ near $x=1$, which may cause the loss of significant digits. Since the values of $1 / \sqrt{1-x^{2}}$ near $x=1$ crucially contribute to the integral, a care is necessary in the example 2 . In fact, we should use

$$
\frac{x^{\prime}(t)}{\sqrt{1-x(t)^{2}}}=\frac{L \pi \cosh (L(t+1) / 2)}{4 \cosh \left(\frac{\pi}{2} \sinh (L(t+1) / 2)\right.},
$$

instead of

$$
\frac{x^{\prime}(t)}{\sqrt{1-x(t)^{2}}}=\frac{L \pi \cosh (L(t+1) / 2)}{4 \cosh ^{2}\left(\frac{\pi}{2} \sinh (L(t+1) / 2)\right) \sqrt{1-\tanh ^{2}\left(\frac{\pi}{2} \sinh (L(t+1) / 2)\right)}} .
$$

The reason the maximum errors do not reach the limit of errors of double precision seems to be, though we are not sure, that the loss of accuracy in the computation of $1 / \sqrt{1-x^{2}}$ near $x=1$ is not eliminated completely.

We now mention the choice of $L$. In order to obtain a good accurate solution, $x(L)$ should be approximately one in theory. Accordingly we would 


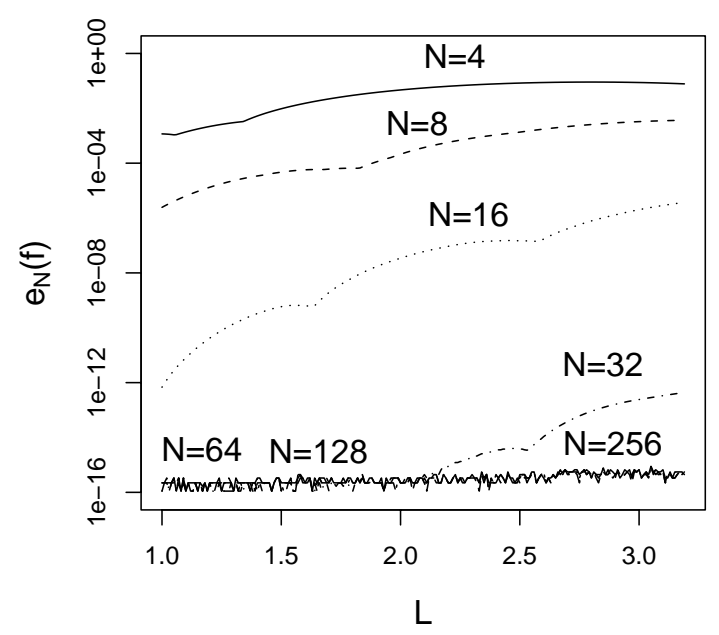

Figure 3. Log plot of the maximum errors for the example 3.

like to choose sufficiently large $L$. However, we must not choose too large L because of the singularity. The computation of the examples 1 and 2 fails if $L>3.2$. So the choice of $L$ should be discussed carefully. Our numerical computation shows that $|1-x(2.0)|=2.25 \times 10^{-5},|1-x(2.5)|=1.11 \times 10^{-8}$, and $|1-x(3.0)|=4.27 \times 10^{-14}$. From this fact and monotonic increase of $x(L)$, we may say that $x(L)$ is approximately one if $L \geq 3.0$. Therefore we can get good results in the examples $1-4$, if we choose $L=3.0$.

We now summarize the advantage and disadvantage of our method. If the integrand is smooth at one end of the interval and singular at another end, then we can compute its indefinite integral with high accuracy by using our method. It is the advantage of our method. However, if the integrand is singular at both ends of the interval, we cannot use our method to compute the indefinite integrals. It is the disadvantage of our method. 


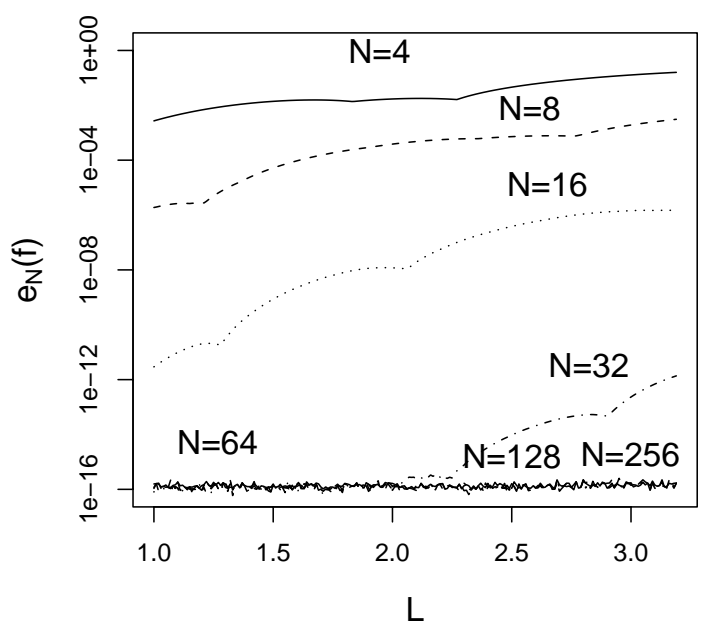

Figure 4. Log plot of the maximum errors for the example 4.

\section{$\S 3 . \quad$ Computation of Serrin's Equations}

\section{§3.1. Discretization of the mapping $\Phi$}

We compute Serrin's equations with the method in the previous section. Let us define a mapping $f \mapsto \Phi[f]$ in the following way:

$$
(\Phi[f])(x)=\int_{0}^{x}\left[-f^{2}(t)+k^{2} \frac{G(t)}{\left(1-t^{2}\right)^{2}}\right] d t .
$$

Here $G$ is defined by (1.3) with $\Omega$ defined in (2.1). Then a solution of Serrin's equations is a fixed point of the mapping $\Phi$.

We now explain the computational procedure, which consists of three steps to discritize (2.1), (1.3), and (3.1). Throughout this section, $N$ denotes $2^{m}$ with a positive integer $m$. We define $x_{j}$ and $w_{j}$ by

$$
x_{j}=x\left(\cos \left(\frac{j \pi}{N}\right)\right), \text { and } w_{j}=x^{\prime}\left(\cos \left(\frac{j \pi}{N}\right)\right),
$$

where $x(t)=\phi(L(t+1) / 2)$ with $\phi$ in (2.4). The weight $\omega_{j}$ is defined by

$$
\omega_{j}= \begin{cases}\frac{\pi}{2 n}, & j=0, \\ \frac{\pi}{n}, & j=1, \ldots, N .\end{cases}
$$


For given $f_{j}$, approximation for $f\left(x_{j}\right)$, the first step is the computation of $\Omega_{j}(j=0, \ldots, N)$, the approximation for $\Omega\left(x_{j}\right)$ :

$$
\begin{aligned}
& a_{j}=\sum_{k=0}^{N} \omega_{k} w_{k} f_{k} T_{j}\left(x_{k}\right), \quad b_{j}=\exp \left(-2 \sum_{k=0}^{N} a_{l} \int_{0}^{x_{j}} T_{k}(t) d t\right), \\
& c_{j}=\sum_{k=0}^{N} \omega_{k} w_{k} b_{k} T_{j}\left(x_{k}\right), \quad d_{j}=\sum_{k=0}^{N} c_{l} \int_{0}^{x_{j}} T_{k}(t) d t, \quad \Omega_{j}=\frac{d_{j}}{d_{0}} .
\end{aligned}
$$

Here, $a_{j}$ and $c_{j}$ are computed by the discrete cosine transform. Then, we define $\Omega_{0}^{\prime}=b_{0} / d_{0}$, which is an approximation for $\Omega^{\prime}(1)$.

The second step is to compute $G$. Note that the indefinite integral in (1.3)

$$
\int_{0}^{x} \frac{t \Omega(t)^{2}}{\left(1-t^{2}\right)^{2}} d t
$$

diverges as $x \rightarrow 1$, which makes it difficult to compute (1.3). Regarding to this, we use L'Hôspital's theorem

$$
\begin{aligned}
& \lim _{x \rightarrow 1}(1-x)^{2} \frac{x \Omega^{2}(x)}{\left(1-x^{2}\right)^{2}}=\lim _{x \rightarrow 1} \frac{x \Omega^{2}(x)}{(1+x)^{2}}=\frac{1}{4}, \\
& \lim _{x \rightarrow 1}(1-x)\left[\frac{x \Omega^{2}(x)}{\left(1-x^{2}\right)^{2}}-\frac{1}{4} \frac{1}{(1-x)^{2}}\right]=\lim _{x \rightarrow 1} \frac{1}{1-x}\left[\frac{x \Omega^{2}(x)}{(1+x)^{2}}-\frac{1}{4}\right] \\
= & -\lim _{x \rightarrow 1} \frac{(1+x)^{2}\left(\Omega^{2}(x)+2 x \Omega(x) \Omega^{\prime}(x)\right)-2 x(1+x) \Omega^{2}(x)}{(1+x)^{4}}=-\frac{\Omega^{\prime}(1)}{2} .
\end{aligned}
$$

Then the integrand of (3.2) satisfies the following asymptotic expantion near $x=1$ :

$$
\frac{x \Omega^{2}(x)}{\left(1-x^{2}\right)^{2}}=\frac{1}{4}(1-x)^{-2}+\frac{\Omega^{\prime}(1)}{2}(1-x)^{-1}+o\left[(1-x)^{-1}\right] .
$$

We therefore obtain

$$
\begin{aligned}
(1- & x)^{2} \int_{0}^{x} \frac{t \Omega^{2}(t)}{\left(1-t^{2}\right)^{2}} d t \\
= & (1-x)^{2} \int_{0}^{x}\left[\frac{t \Omega^{2}(t)}{\left(1-t^{2}\right)^{2}}-\frac{1}{4}(1-t)^{-2}+\frac{\Omega^{\prime}(1)}{2}(1-t)^{-1}\right] \\
& +\frac{1}{4}(1-x)^{2} \int_{0}^{x} \frac{d t}{(1-t)^{2}}-\frac{\Omega^{\prime}(1)}{2}(1-x)^{2} \int_{0}^{x} \frac{d t}{1-t} \\
= & (1-x)^{2} \int_{0}^{x} g(t) d t+\frac{1}{4}(1-x)+\frac{\Omega^{\prime}(1)}{2}(1-x)^{2} \log (1-x),
\end{aligned}
$$


where we set

$$
g(x)=\frac{t \Omega^{2}(t)}{\left(1-t^{2}\right)^{2}}-\frac{1}{4}(1-t)^{-2}+\frac{\Omega^{\prime}(1)}{2}(1-t)^{-1} .
$$

Here, $g(x)$ is integrable near $x=1$ and is much less singular than the integrand of (3.2). Therefore, we apply the method in Section 3 to an indefinite integral of $g(x)$ to compute $(3.2)$.

Keeping these facts in mind, we define $G_{j}$, which is the approximation for $G\left(x_{j}\right)$ as follows:

$$
\begin{aligned}
& g_{j}=\frac{x_{j} \Omega_{j}^{2}}{\left(1-x_{j}^{2}\right)^{2}}-\frac{1}{4\left(1-x_{j}\right)^{2}}-\frac{\Omega_{0}^{\prime}}{2\left(1-x_{j}\right)} \\
& p_{j}=\sum_{k=0}^{N} \omega_{k} w_{k} g_{k} T_{j}\left(x_{k}\right), \quad q_{j}=\sum_{k=0}^{N} p_{k} \int_{0}^{x_{k}} T_{j}(t) d t \\
& G_{j}^{(1)}=2\left(1-x_{j}\right)^{2} q_{j}+\frac{1}{2}\left(1-x_{j}\right)+\Omega_{0}^{\prime}\left(1-x_{j}\right)^{2} \log \left(1-x_{j}\right), \\
& r_{j}=\sum_{k=0}^{N} \omega_{k} w_{k} \frac{\Omega_{k}^{2}}{\left(1+x_{k}\right)^{2}} T_{j}\left(x_{k}\right) \\
& G_{j}^{(2)}=2 x_{j} \sum_{k=0}^{N} r_{k} \int_{0}^{x_{k}} T_{j}(t) d t \\
& G_{j}=G_{j}^{(1)}+G_{j}^{(2)}-P\left(x_{j}-x_{j}^{2}\right) .
\end{aligned}
$$

The final step is the computation of (3.1). To deal with the singularity of the integrand in the right hand side of (3.1), we recall the two lemmas in Serrin [9].

Lemma 3.1. $\quad$ Let $G$ be defined by (1.3), where $f$ and $\Omega$ are solutions of Serrin's equations. Then $G(1)=0$, and $G^{\prime}(1)=P-1$.

Lemma 3.2. Let $f$ be a solution of Serrin's equations. Then,

$$
f(x) \sim O\left(\log \frac{1}{1-x}\right) \quad \text { as } \quad x \rightarrow 1 .
$$

From Lemma 3.2, we see that $f^{2}(t)$ is integrable. On the other hand, the second term $G(t) /\left(1-t^{2}\right)^{2}$ is not integrable. With Lemma 3.1 and L'Hôpital's theorem, we have

$$
k^{2} \frac{G(x)}{\left(1-x^{2}\right)^{2}}=\frac{1}{4} k^{2}(1-P) \frac{1}{1-x}+o\left(\frac{1}{1-x}\right) .
$$


Therefore,

$$
\begin{aligned}
f(x) & =\int_{0}^{x}\left[-f^{2}(t)+k^{2} \frac{G(t)}{\left(1-t^{2}\right)^{2}}\right] d t \\
& =\int_{0}^{x} h(t) d t+\frac{1}{4} k^{2}(1-P) \log \left(\frac{1}{1-x}\right),
\end{aligned}
$$

where

$$
h(t)=-f^{2}(t)+k^{2} \frac{G(t)}{\left(1-t^{2}\right)^{2}}-\frac{1}{4} k^{2}(1-P) \frac{1}{1-t} .
$$

Keeping these facts in mind, we define $\Phi[f]_{j}$ (approximation for $\Phi[f]\left(x_{j}\right)$ ) as follows:

$$
\begin{aligned}
& h_{j}=-f_{j}^{2}+k^{2} \frac{G_{j}}{\left(1-x_{j}^{2}\right)^{2}}-\frac{1}{4} k^{2}(1-P) \frac{1}{1-x_{j}}, \quad s_{j}=\sum_{k=0}^{N} \omega_{k} w_{k} h_{k} T_{j}\left(x_{k}\right), \\
& \Phi[f]_{j}=\sum_{l=0}^{N} s_{l} \int_{0}^{x_{j}} T_{l}(t) d t+\frac{1}{4} k^{2}(1-P) \log \left(\frac{1}{1-x_{j}}\right) .
\end{aligned}
$$

\section{§3.2. Tracing the solution path with pseudoarclength method}

In this subsection, we numerically solve $f=\Phi[f]$ by the pseudoarclength method. As for the pseudoarclength method, see [1, 2, 4]. It is one of the methods to trace a solution path with critical points. Note that Serrin's equations have an exact solution $f(x)=0, \Omega(x)=x$ for the parameter $(k, P)=(0,0)$. Starting from this trivial solution for $(k, P)=(0,0)$, we can compute solutions for arbitrary parameters $(k, P)$. The computation of solution for $(k, P)$ consists of two steps. In the first step, we compute a solution at $(k, 0)$ starting from the trivial solution for $(k, P)=(0,0)$ with a generalized Newton's method. In the next step, we compute a solution at $(k, P)$ starting from the solution at $(k, 0)$ by the pseudoarclength method. Specifically, let $\left\{f_{j}\right\}_{j=0}^{N}$ be a solution at $(k, P)=\left(k_{0}, 0\right)$. Then, for small $\Delta k$, we compute a solution at $(k, P)=\left(k_{0}+\Delta k, 0\right)$ with a generalized Newton's method for the equation $f-\Phi[f]=0$ with the initial value $\left\{f_{j}\right\}_{j=0}^{N}$. With this process starting from $(k, P)=(0,0)$, solutions can be found at $(k, P)=(k, 0)$ for arbitrary $k \geq 0$. In the generalized Newton's method, we use the following approximate Jacobian of $\Phi$.

$$
D \Phi[f]=\left(D \Phi_{i j}\right)_{i, j=0, \ldots, N}, \quad D \Phi_{i j}=\frac{\Phi\left[f+\varepsilon_{j}\right]_{i}-\Phi[f]_{i}}{\varepsilon}
$$


where

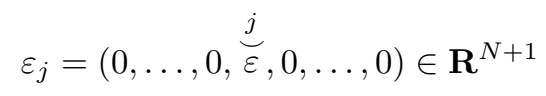

and $\varepsilon$ is a small number. In our computation, $\varepsilon=1.0 \times 10^{-6}$ was used.

Next, starting from a solution at $(k, P)=\left(k_{0}, 0\right)$, which is computed by the above method, we compute a solution at $(k, P)=\left(k_{0}, P\right)$ by using the pseudoarclength method for $P$.

Figure 5 is the solution path when $k=10$ in $0 \leq \Omega^{\prime}(1) \leq 0.2$. We set $L=2.4$ and $N=256$. In this case, $1-x(1) \approx 6.97 \times 10^{-8}$. From Figure 5 , we see that the solution path has a turning point at $P^{*} \approx 0.478$. Figure 6 is the same solution path as Figure 5 in $0 \leq \Omega^{\prime}(1) \leq 400$. From Figure 6 , we see that the solution path rapidly increases and tends to infinity as $P \rightarrow P^{* *}$, which lies between 0 and 0.1 .

Figures 7 and 8 show the graphs of the solution at $(k, P)=(10,0.0832)$ which lies on the upper branch of Figure 5. The left figure shows the graph of $f$ and the right one shows that of $\Omega$. From these graphs we see the following properties. The function $\Omega(x)$ is very small except in a small neighborhood of $x=1$ and increases to one very quickly near $x=1$. On the other hand, as $x \rightarrow 1, f$ swings rapidly among negative and positive values. It implies that there is a strong downward jet near the vortex filament.

Although $f \sim|\log (1-x)|$ diverges at $x=1$, the divergence is very weak. Accordingly, the divergence of $f$ at $x=1$ is difficult to trace numerically. For example, $f$ seems to be monotone decreasing when $P$ is smaller than 0.0766 (see Figure 8), although it eventually tends to $+\infty$. This phenomenon is also caused by the same reason. (Figure 9 also shows the limitation of our method.)

Near $(k, P)=(10,0.0766)$, the upper branch of solution path has rapid growth as is seen from Figure 6 . Actually, at $(k, P)=(10,0.0766), \Omega^{\prime}(1)=$ $1.23 \times 10^{4}$ and the gradient of the solution path is approximately $-1.09 \times 10^{8}$. From these observation, we can expect that there exists a $P^{* *} \approx 0.0766$, such that $\Omega^{\prime}(1)$ tends to infinity as $P$ tends to $P^{* *}$ through the upper branch of the solution path at $k=10$. As for the number of solutions, we may say that when $k=10$, Serrin's equations have one solution for $0 \leq P \leq P^{* *}$, two solutions for $P^{* *}<P<P^{*}$, one for $P=P^{*}$, and none for $P^{*}<P$.

The solution path has a qualitative difference if $k$ varies. Figure 10 shows the solution path at $k=3$, where no turning point exists. It suggests that there is a critical value $k^{*}$ between $k=3$ and $k=10$ such that a picture like Figure 5 is observed for $k>k^{*}$ and that like Figure 10 for $k<k^{*}$.

To verify whether this expression is true, we compute the solution paths 


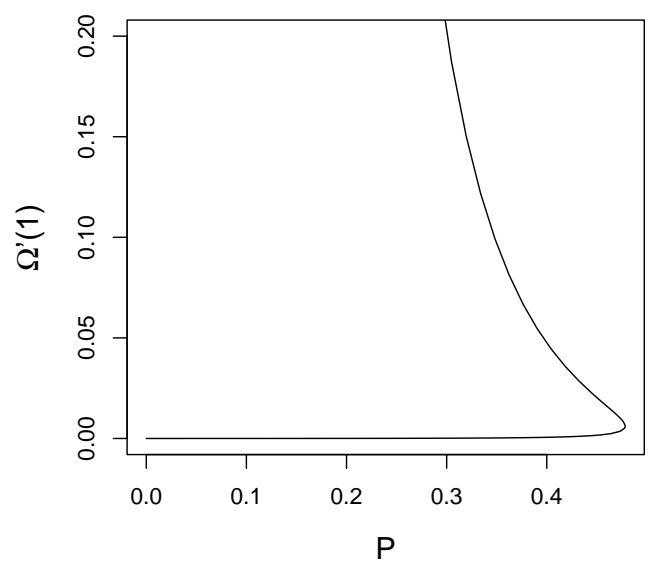

Figure 5. The solution path at $k=10,\left(0 \leq \Omega^{\prime}(1) \leq 0.2\right)$.

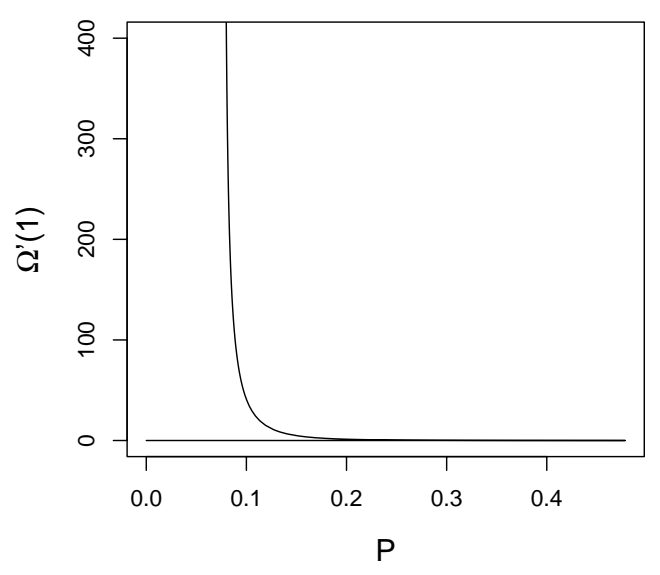

Figure 6 . The solution path at $k=10 .(L=2.4, N=256)$. 


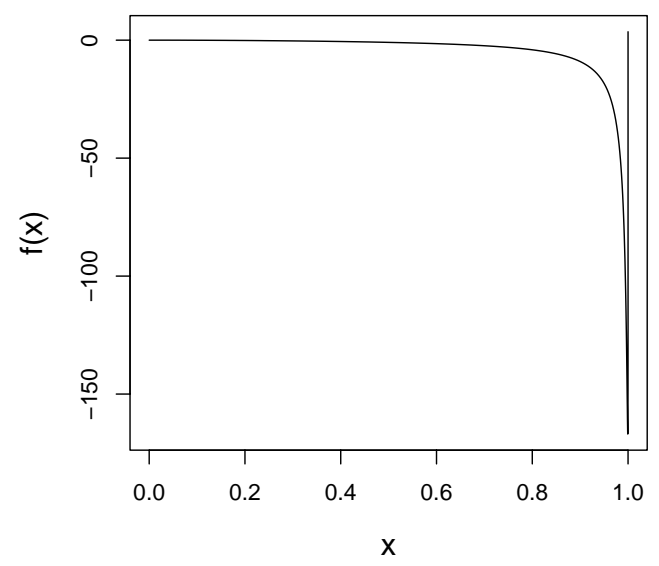

Figure 7. The graph of a solution $f$ at $(k, P)=(10,0.0832),(L=2.4, N=256)$.

for $k$ from $k=1$ to 10 . We carried out these computations until $\Omega^{\prime}(1)$ became greater than $1.00 \times 10^{4}$. Figure 10 shows the turning points and the points at which $\Omega^{\prime}(1)$ first becomes greater than $1.00 \times 10^{4}$. From Figure 11 we see that the turning point exists for $k>k^{*}$, where $k^{*}$ is somewhere between 3 and 4 . In order to determine the precise value of $k^{*}$, we used the bisection method. We found that $k^{*}$ is approximately equal to 3.4306 . We also know that the all the points at which $\Omega^{\prime}(1) \rightarrow \infty$ lies near the curve $P k^{2}=7.6478$. Therefore we can expect that $P k^{2}=7.6478$ is the critical value where Serrin's equations lose the uniqueness of the solution. Note that Goldshtik \& Shtern [3] claimed numerically that the critical value for the lack of the uniqueness of solutions was $P k^{2}=7.6447$. Our result $P k^{2}=7.6478$ is very close to their result. But we do not know which is a better value.

\section{$\S 4$. Accuracy of Our Numerical Scheme}

The parameters which affect the accuracy of our method are the truncation $L$ and the number of collocation points $N$. Accordingly we check the dependence of the error on these parameters.

We define the following quantities, which we call the relative error of $f$ and $\Omega$, respectively: 


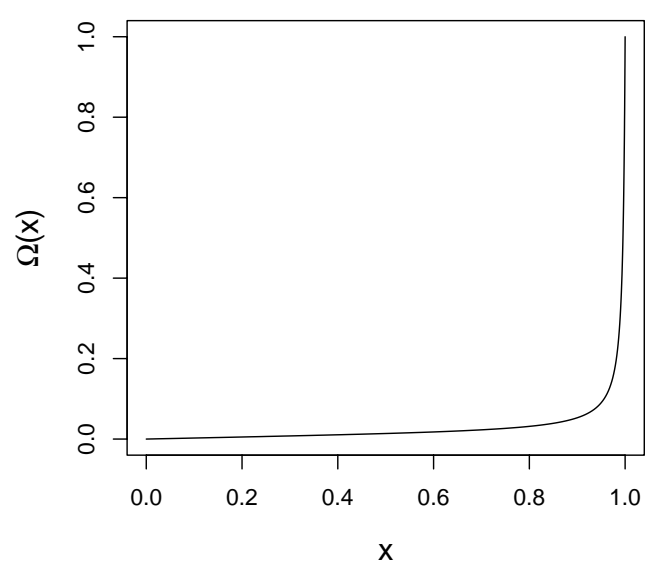

Figure 8. The graph of a solution $\Omega$ at $(k, P)=(10,0.0832),(L=2.4, N=256)$.

$$
\begin{aligned}
& E_{N}(f)=\frac{\max _{0 \leq j \leq N}\left|f_{j}^{(N)}-f_{2 j}^{(2 N)}\right|}{\max _{0 \leq j \leq N}\left|f_{j}^{(N)}\right|}, \\
& E_{N}(\Omega)=\frac{\max _{0 \leq j \leq N}\left|\Omega_{j}^{(N)}-\Omega_{2 j}^{(2 N)}\right|}{\max _{0 \leq j \leq N}\left|\Omega_{j}^{(N)}\right|},
\end{aligned}
$$

where $\left\{f_{j}^{(n)}\right\}_{j=0}^{n}$ and $\left\{\Omega_{j}^{(n)}\right\}_{j=0}^{n}$ are numerical solutions with the number of points $n$. Figures 12 and 13 show the relative errors of $f$ and $\Omega$ at $(k, P)=$ $(5,0.3)$ with various $L$ and $N$. The notation $8-16$ implies the graph of relative error between the numerical solution with $N=8$ and $N=16$, i.e., $E_{8}(f)$ and $E_{8}(\Omega)$. We find that the relative errors of $f$ and $\Omega$ rapidly decrease as $N$ increases when $L$ is fixed. But the relative errors of $f$ at $64-128,128-256$, and 256-512 are between $1.00 \times 10^{-9}$ and $1.00 \times 10^{-14}$. On the contrary, the corresponding relative errors of $\Omega$ decrease and tend to $1.00 \times 10^{-16}$, which are the machine precision, for sufficiently large $L$. The reason why the relative error of $f$ hardly improve before it reaches the limits of errors of double precision is that the singularity of $f$ at $x=1$ is not completely resolved.

We also see that for a fixed $N$ the relative errors increase as $L$ increases and the computation fails when $L>2.6$, as was explained in the previous 


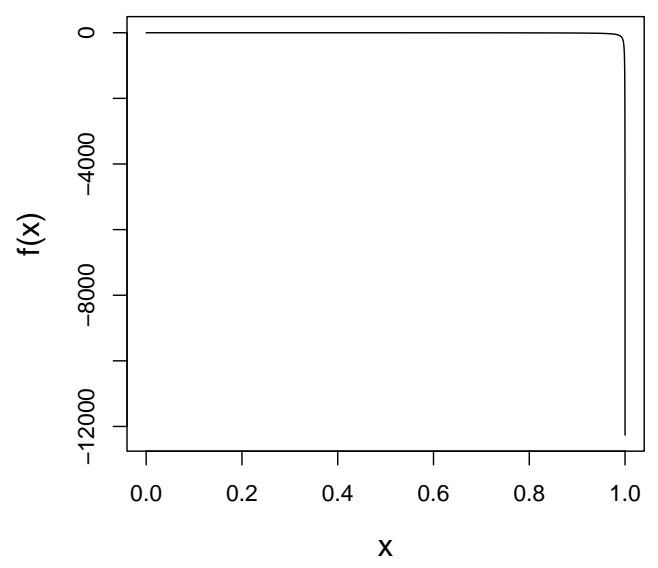

Figure 9. The graph of a solution $f$ at $(k, P)=(10,0.0766) .(L=2.4, N=256)$.

section. More precisely, the maximum of $L$ where the computation succeeds gets larger as $N$ increases. Note that there are cuts in the graph of 8-16 in Figures 12 and 13. These cuts mean that the Newton's iteration fails for the corresponding parameters $N$ and $L$ are corresponding values. There is no cut in the other graphs in Figures 12 and 13. While the computation for $N=256$ and $L=2.6$ works well in the case of $(k, P)=(5,0.3)$, the Newton method did not converge with $N=256$ and $L=2.6$ in the other case of $(k, P)=(10,0.0766)$. Taking account of these observations, we chose $L=2.4$ and $N=256$ in our computation.

\section{$\S 5 . \quad$ Estimate on the Parameter for the Existence of Solutions}

Serrin proved the following lemma [9].

Lemma 5.1. Consider the following equations with a parameter $\lambda$

$$
f^{\prime}(x)+f^{2}(x)=-\lambda \frac{x}{(1+x)^{2}(1-x)}, \quad(0 \leq x<1)
$$

and a corresponding condition

$$
f(0)=0 .
$$




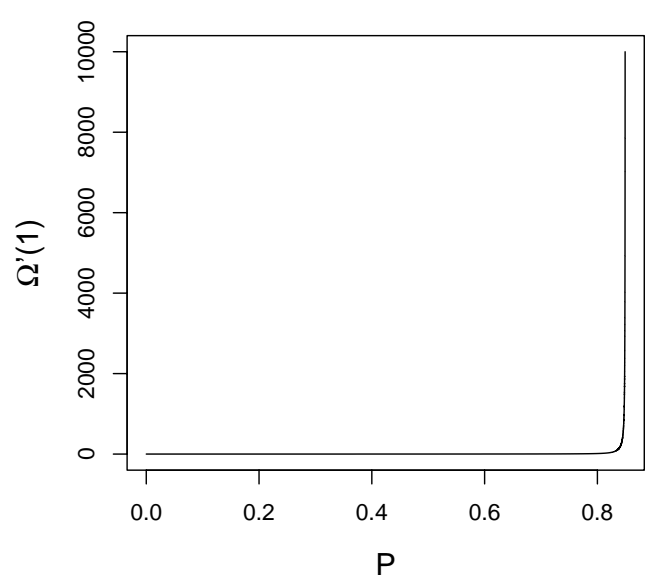

Figure 10. The solution path at $k=3$.

If the equation (5.1) with (5.2) has a solution, which exists on the entire $[0,1)$ and satisfy $f(x)=O\left(\log \left[(1-x)^{-1}\right]\right)$ as $x \rightarrow 1$, for some $\lambda>0$, then Serrin's equations (1.1)-(1.4) has a solution for all $P \geq 0$ and $k \geq 0$ satisfying $P k^{2}<\lambda$.

We now compute the critical value $\lambda=\lambda^{*}$, at which a solution of (5.1) and (5.2) exists. First of all, we transform (5.1) and (5.2) to the following integral equation:

$$
f(x)=\int_{0}^{x}\left[-f^{2}(t)-\lambda \frac{t}{(1+t)^{2}(1-t)}\right] d t .
$$

Note that the first term $-f^{2}(t)$ is integrable in $[0,1]$ as long as $f(t)=O[\log (1-$ $x)$ ], while the second term of integrand in (5.3) diverges as $x \rightarrow 1$. Actually, we have

$$
\int_{0}^{x} \frac{t}{(1+t)^{2}(1-x)} d t=\frac{1}{4}\left[\log \frac{1+x}{1-x}+\frac{2}{1+x}\right] .
$$

We therefore obtain

$$
f(x)=-\int_{0}^{x} f^{2}(t) d t-\frac{\lambda}{4}\left[\log \frac{1+x}{1-x}+\frac{2}{1+x}\right],
$$

to which we can apply our method. 


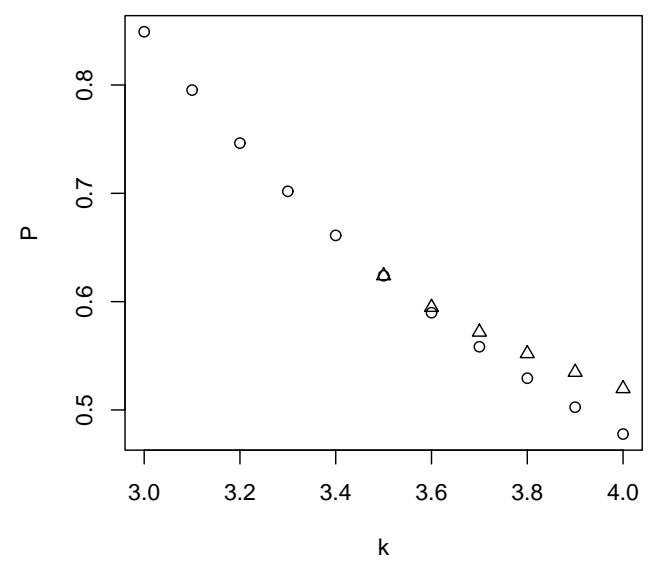

Figure 11. The plot of the turning points (triangles) and the points at which $\Omega^{\prime}(1) \approx \infty$ (circles) for $k=3.0,3.1, \ldots, 4.0$.

Now we define a mapping $f \mapsto \Psi[f]$ as follows:

$$
\Psi[f](x)=-\int_{0}^{x} f^{2}(t) d t-\frac{\lambda}{4}\left[\log \frac{1+x}{1-x}+\frac{2}{1+x}\right] .
$$

Because a solution of (5.3) is a fixed point of $\Psi$, it is sufficient to determine $\lambda^{*}$ so that $\Psi$ has a fixed point for $\lambda<\lambda^{*}$.

Note that $f=0$ is a solution of (5.3) for $\lambda=0$. By increasing $\lambda$, we compute $(f, \lambda)$ with the Newton method. If the Newton iteration does not converge at $\lambda=\lambda_{0}+\Delta \lambda$, we halve the increment value and try the computation again. If the iterations no longer converge for $\Delta \lambda>\varepsilon_{\Delta}, \varepsilon_{\Delta}=1.0 \times 10^{-8}$ in our computation, we stop the computation and take $\lambda$ as the approximation of the critical value $\lambda^{*}$. We also note that (5.1) and (5.2) can have only one at most solution for a given $\lambda$ by the uniqueness of the solution of ordinary differential equations. Accordingly, the solution path of (5.1) and (5.2) never has a turning point. Therefore (5.1) and (5.2) have no solution for $\lambda>\lambda^{*}$, which is computed by the above method.

Table 1 shows the results of the computation of $\lambda^{*}$ at $N=16,32,64,128$, and 256. Third column of Table 9 shows values of $\left|\lambda_{N / 2}^{*}-\lambda_{N}^{*}\right|$, where $\lambda_{N}^{*}$ is defined as the approximate value of $\lambda^{*}$ at $N$. It shows that the critical value 


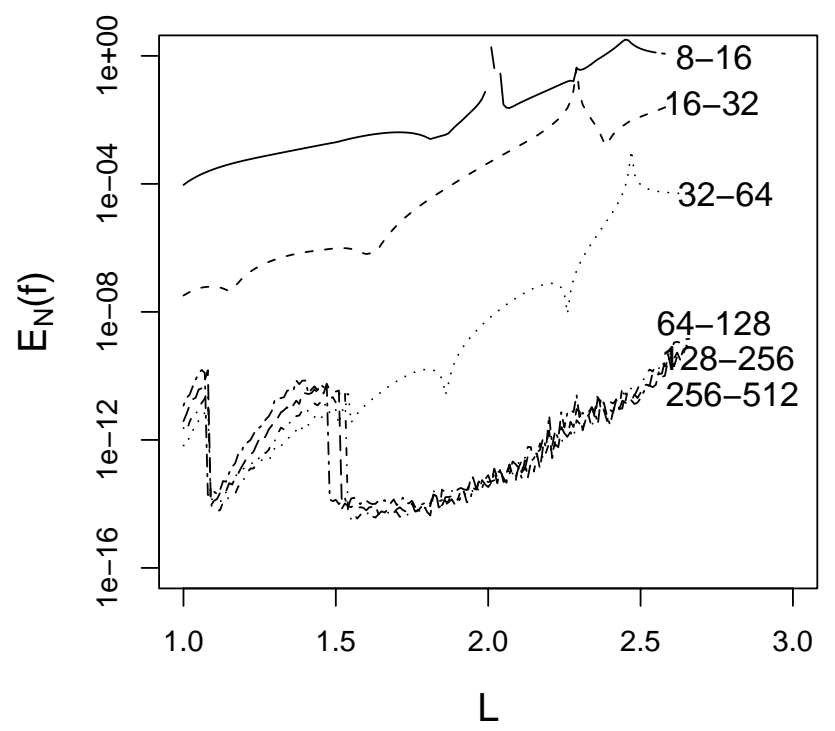

Figure 12. Graph of the relative error of $f$.

Table 1. Results of $\lambda^{*}$ and $\left|\lambda_{N / 2}^{*}-\lambda_{N}^{*}\right|$.

\begin{tabular}{r|c|c}
\multicolumn{1}{c|}{$N$} & $\lambda^{*}$ & $\left|\lambda_{N / 2}^{*}-\lambda_{N}^{*}\right|$ \\
\hline 16 & 7.49 & - \\
32 & 7.63 & $1.43 \times 10^{-1}$ \\
64 & 7.64 & $4.99 \times 10^{-3}$ \\
128 & 7.63 & $2.57 \times 10^{-3}$ \\
256 & 7.63 & $8.52 \times 10^{-4}$
\end{tabular}

$\lambda^{*}$ is approximately 7.63 .

In [9], Serrin computed numerically $\lambda^{*}$, and concluded that $\lambda^{*}$ was approximately 8.12 . However since Serrin did not show what kind of numerical scheme he had used, we cannot follow his computation. So, let alone examine the accuracy; we compute the critical value of $\lambda$, which is denoted by $\lambda^{*}$, with 


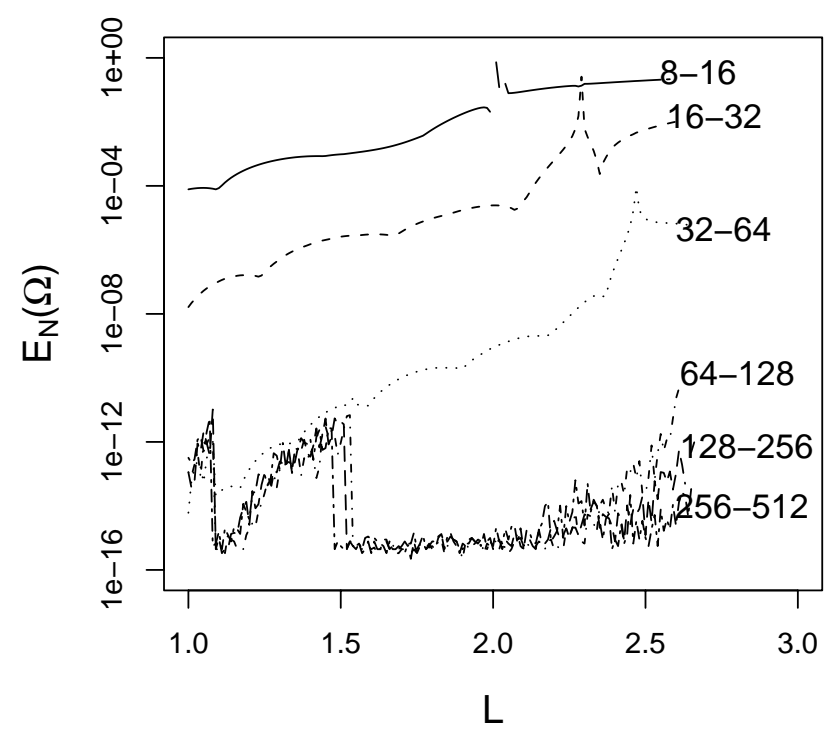

Figure 13. The relative error of $\Omega$ v.s. $L$.

our method and examine the validity of Serrin's value.

Our result $\lambda^{*} \approx 7.63$ is substantially different from the Serrin's result $\lambda^{*} \approx 8.12$. It suggests that Serrin's value may be doubtful. Note that our value 7.63 is close to the lack of the uniqueness of solutions of Serrin's equations $k^{2} P=7.65$. It suggests that the existence of solutions of $(5.1)$ and (5.2) is related to the uniqueness of the solution of Serrin's equations. But the proof of the above claim is left for the future work.

\section{$\S 6 . \quad$ Conclusion}

We proposed a numerical method for indefinite integrals whose integrands had a singularity at one side of the interval using the double exponential transformation and the Chebyshev expansion. With the numerical method, we solved Serrin's equations, and we verified that there existed a region of $(k, P)$ where Serrin's equations had two solutions. We also confirmed that near the boundary of the region where Serrin's equations had two solutions there exists 
a solution that had quick change near the $x=1$. This change was the following: $f$ swung from a large negative value to the positive infinity as $x \rightarrow 1$, and $\Omega$ increased to one. It physically means there is a strong downward jet near the vortex filament. We also computed the value of sufficient condition for the existence of solutions of Serrin's equation, and we found that Serrin's result $P k^{2} \leq 8.12$ was not optimal.

In our method, we used "one-side" double exponential transformation $\phi(t)$, which means that $\phi$ maps $[0, \infty)$ to $[0,1)$ and $\left|f(\phi(t)) \phi^{\prime}(t)\right|$ decays double exponentially only as $t \rightarrow+\infty$. We usually use the double exponential transformation $\psi(t)$ such that $\psi$ maps $(-\infty, \infty)$ to $(-1,1)$ and $\left|f(\psi(t)) \psi^{\prime}(t)\right|$ decays double exponentially as $t \rightarrow \pm \infty$. However, we know that our method works better than "two-side" DE for indefinite integrals whose integrands has a singularity at one side of the interval. Actually, Table 2 shows the maximum errors of the computations of the examples 1 in Section 2 with one-sided and two-sided double exponential transformations, from which we see that the computation of the one-sided DE transformation when $N=n$ is almost the same accuracy as that of the two-sided DE transformation when $N=2 n$.

Table 2. One-sided DE and two-sided DE errors of example $1(L=3.0)$.

\begin{tabular}{c|cc}
$N$ & one-side & two-side \\
\hline 4 & $6.70 \times 10^{-2}$ & $4.99 \times 10^{-1}$ \\
8 & $3.74 \times 10^{-3}$ & $1.38 \times 10^{-1}$ \\
16 & $1.85 \times 10^{-6}$ & $5.00 \times 10^{-3}$ \\
32 & $4.74 \times 10^{-13}$ & $3.21 \times 10^{-6}$ \\
64 & $1.55 \times 10^{-15}$ & $7.45 \times 10^{-13}$ \\
128 & $1.66 \times 10^{-15}$ & $1.44 \times 10^{-15}$ \\
256 & $1.77 \times 10^{-15}$ & $1.55 \times 10^{-15}$
\end{tabular}

However, there is a possibility that the two-sided DE transformation using the sinc interpolation $[6,11]$ or the fast Fourier transform (FFT) [5] would be better. In order to judge which methods are better, we have to execute the rigorous error estimate for our method, which is left for another future work.

\section{Acknowledgements}

The author would like to thank Professor Hisashi Okamoto for his helpful suggestion, advice and continuous encouragement. The author also thanks Dr. 
Takuya Ooura and Professor Masaharu Nagayama for numerous advices about numerical computations.

\section{References}

[1] E. Doedel, H. B. Keller and J.-P. Kernévez, Numerical analysis and control of bifurcation problems. I. Bifurcation in finite dimensions, Internat. J. Bifur. Chaos Appl. Sci. Engrg. 1 (1991), no. 3, 493-520.

[2] $ـ$ Numerical analysis and control of bifurcation problems. II. Bifurcation in infinite dimensions, Internat. J. Bifur. Chaos Appl. Sci. Engrg. 1 (1991), no. 4, 745-772.

[3] M. A. Goldshtik and V. N. Shtern, Collapse in conical viscous flows, J. Fluid Mech. 218 (1990), 483-508.

[4] H. B. Keller, Numerical solution of bifurcation and nonlinear eigenvalue problems, in Applications of bifurcation theory (Proc. Advanced Sem., Univ. Wisconsin, Madison, Wis., 1976), 359-384. Publ. Math. Res. Center, 38, Academic Press, New York.

[5] K. Kobayashi, H. Okamoto and J. Zhu, Numerical computation of water and solitary waves by the double exponential transform, J. Comput. Appl. Math. 152 (2003), no. 1-2, 229-241.

[6] M. Mori and M. Muhammad, Numerical indefinite integration by the double exponential transformation, Trans. of the Japan Soc. for Industrial and Appl. Math., 13 (2003), no. 3, 361-366 in Japanese.

[7] A. Nurmuhammad, M. Muhammad and M. Mori, Numerical solution of initial value problems based on the double exponential transformation, Publ. Res. Inst. Math. Sci. 41 (2005), no. 4, 937-948.

[8] M. Sugihara, Optimality of the double exponential formula-functional analysis approach, Numer. Math. 75 (1997), no. 3, 379-395.

[9] J. Serrin, The swirling vortex, Phil. Trans. R. Soc. Lond. A, 271 (1972), 325-260.

[10] H. Takahasi and M. Mori, Double exponential formulas for numerical integration, Publ. Res. Inst. Math. Sci. 9 (1973/74), 721-741.

[11] K. Tanaka, M. Sugihara and K. Murota, Numerical indefinite integration by double exponential sinc method, Math. Comp. 74 (2005), no. 250, 655-679 (electronic). 